\title{
Análisis estadístico del espesor de recubrimiento de sistemas de protección dúplex durante el ensayo climático acelerado de humedad y temperatura controlada
}

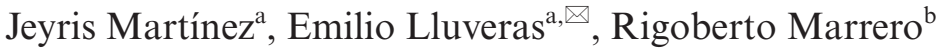 \\ ${ }^{a}$ Laboratorio de Ensayos de Tropicalización LABET, Carretera del Morro 7246, Km. 2 1⁄2, Cojímar, La Habana, Cuba \\ bUniversidad Tecnológica de La Habana "José Antonio Echeverría”, Calle 114 \# 11901 / Ciclovía y Rotonda CP 19390, \\ Marianao, La Habana, Cuba \\ ( ${ }^{\bowtie}$ Autor para la correspondencia: jeyris@nauta.cu; jeyris@1abet.ctec.cu)
}

Enviado: 26 Septiembre 2018; Aceptado: 27 Marzo 2019; Publicado on-Line: 1 Julio 2019

\begin{abstract}
RESUMEN: El acero es un material metálico muy versátil y ampliamente utilizado en la vida moderna. Desafortunadamente, este material está propenso al fenómeno de la corrosión y el pasar del tiempo, puede contribuir a la falla del producto. Para lograr minimizar este proceso se utilizan diferentes tipos de recubrimientos, debido a su eficacia, economía y facilidad de aplicación. La degradación de la película aplicada sobre el acero por los agentes atmosféricos es resultado de la combinación de diversos factores ambientales, debido a las variaciones cíclicas altamente destructivas. El presente trabajo pretende determinar la existencia de diferencias estadísticas significativas en las distribuciones del espesor de recubrimiento en sistemas dúplex (Fe/Zn/Pintura), antes y después de realizado el ensayo de humedad y temperatura con condensación en atmósfera constante, a partir de diferentes pruebas realizadas en el software Statgraphics Centurion. Se demostró la influencia de las condiciones prefijadas sobre el espesor de recubrimiento antes y después de realizado el ensayo. Dichos valores se encontraron en un intervalo adecuado para garantizar la resistencia a la corrosión, no evidenciándose deterioro visible en la superficie de las probetas analizadas.
\end{abstract}

PALABRAS CLAVE: Ensayo; Espesor; Estadística; Protección dúplex

Citar como/Citation: Martínez, J.; Lluveras, E.; Marrero, R. (2019). "Análisis estadístico del espesor de recubrimiento de sistemas de protección dúplex durante el ensayo climático acelerado de humedad y temperatura controlada". Rev. Metal. 55(2): e146. https://doi.org/10.3989/revmetalm.146

\begin{abstract}
Statistical analysis of the coating thickness of duplex protection systems during the accelerated climatic test of humidity and controlled temperature. Steel is a very versatile metallic material and widely used in modern life. Unfortunately, this material is prone to the phenomenon of corrosion and over time, can contribute to the failure of the product. In order to minimize this process, different types of coatings are used, due to their efficiency, economy and ease of application. The degradation of the film applied to steel by atmospheric agents is the result of the combination of various environmental factors, due to the highly destructive cyclic variations. The present work intends to determine the existence of significant statistical differences in the thickness distribution of coating in duplex systems (Fe/Zn/Paint), before and after conducting the humidity and temperature test with condensation in a constant atmosphere, from different tests carried out in the Statgraphics Centurion software. The influence of the preset conditions on the coating thickness before and after the test was demonstrated was demonstrated. These values were found in a suitable interval to guarantee the resistance to corrosion, not showing visible deterioration in the surface of the analyzed specimens.
\end{abstract}

KEYWORDS: Duplex protection; Statistics; Test; Thickness

ORCID: Jeyris Martínez Gutiérrez (https://orcid.org/0000-0002-0564-8661); Emilio Miguel Lluveras Pérez (https:// orcid.org/0000-0002-3648-6204); Rigoberto Marrero Águila (https://orcid.org/0000-0002-3011-5701)

Copyright: (C) 2019 CSIC. Este es un artículo de acceso abierto distribuido bajo los términos de la licencia de uso y distribución Creative Commons Reconocimiento 4.0 Internacional (CC BY 4.0). 


\section{INTRODUCCIÓN}

Las acciones ambientales para un desarrollo sostenible se basan en los requerimientos del desarrollo económico y social del país. Los recursos naturales deben aprovecharse de manera racional, previniendo la generación de impactos negativos sobre el medio ambiente (CITMA, 1997).

Uno de los procesos naturales a los que nos enfrentamos todos y cada uno de los seres que coexistimos en este planeta, sean vivos o no, es el deterioro debido a la interacción con el medio ambiente. En la naturaleza, los metales están presentes en forma de minerales en conjunto con otros elementos como el oxígeno, nitrógeno, carbono, entre otros. El hombre tiene la necesidad de separarlos de estos elementos de manera tal que sea posible utilizarlos. A medida que la energía requerida para realizar esta tarea aumenta, el proceso en el cual el metal regresa a su estado natural también lo hace (Vásquez Rodríguez, 2013).

La expansión de las industrias, así como el constante crecimiento de las ciudades y el continuo avance de la civilización, han contribuido a una mayor contaminación atmosférica, provocando entre otros efectos indeseables, el deterioro acelerado de los materiales (Marrero-Águila et al., 2013).

El acero, en forma de lámina, es un producto muy versátil. Se suministra en muchas formas y tamaños, y es empleado con múltiples fines incluyendo construcciones de acero, paneles de vehículos automotrices, señales, y equipos electrodomésticos. El bajo costo, alta resistencia mecánica y su conformabilidad de la lámina de acero, son algunas razones de su amplio uso.

Desafortunadamente, muchos productos fabricados de acero, están propensos a la corrosión, un fenómeno que ocasiona una superficie desagradable y, al pasar el tiempo, puede contribuir a la falla del producto. Por esta razón, el acero debe estar protegido por una variedad métodos que van desde aleación interna (por ejemplo, aceros inoxidables) hasta recubrimientos de pintura o recubrimientos metálicos (Vásquez Rodríguez, 2013).

Los sistemas de protección secundaria (a base de recubrimientos) constituyen sin dudas el método más empleado en el combate contra el deterioro en las estructuras. La razón de ello es la eficacia, economía y facilidad de aplicación. Los sistemas de protección y su forma de aplicación deben garantizar que las estructuras con un elevado nivel de deterioro recuperen las prestaciones exigidas durante su construcción, tales como: estética, funcionalidad $\mathrm{y}$, sobre todo, seguridad. Deben poseer entre sus características fundamentales, buena adherencia, dureza, baja porosidad y uniformidad en el espesor (Castañeda et al., 2012).

Cutipa Mamani (2014), afirma que los sistemas de protección dúplex formado por $(\mathrm{Fe} / \mathrm{Zn} /$
Pintura) garantiza la protección del acero contra la corrosión. Los recubrimientos de pintura poseen normalmente poros y microgrietas que permiten el paso de la humedad ambiental. Si estos recubrimientos se aplican directamente sobre la superficie del acero, el óxido de hierro que se forma inicialmente debajo de la capa de pintura tiende a agrandar estas grietas, por ser voluminoso y por tanto, facilita la penetración de los agentes agresivos hacia la superficie del acero, con la consiguiente formación de nuevas cantidades de óxido y ampollamiento de la pintura. La formación de productos de corrosión de zinc compactos y adherentes rellenan las grietas, prolongando la duración de la película de pintura. Este comportamiento trae como consecuencia que los sistemas dúplex presenten un tiempo de vida útil mayor que el obtenido en cada sistema por separado.

Las pinturas actúan normalmente como barrera física entre el acero y la atmósfera corrosiva. Para ejercer con eficacia esta acción su espesor no debería situarse nunca por debajo de un determinado límite, variable con el tipo de pintura, estado de la superficie, severidad de las condiciones ambientales y tiempo previsto de exposición. La durabilidad de la misma se relaciona estrechamente con el espesor aplicado (Simancas Peco, 1992).

Los recubrimientos de pintura expuestos a la atmósfera experimentan con el tiempo una degradación progresiva que conduce a una pérdida completa de sus propiedades de protección. El conocimiento de los mecanismos de degradación atmosférica de los sistemas de pintura y su durabilidad exigen una experimentación en campo, lo que supone el gran inconveniente de la lentitud de respuesta de este tipo de ensayos. Para contrarrestar este gran inconveniente se acostumbra a realizar ensayos de corta duración (Simancas y Morcillo, 1998).

En la práctica, es muy útil conocer la resistencia a la corrosión de un determinado metal o aleación en un medio ambiente definido y/o bajo una condición o servicio específico. Por ello, las pruebas de corrosión son muy importantes al permitir la evaluación y selección de un material, la estimación de vida útil del mismo y la determinación de los medios más económicos de reducción de la corrosión (Mina Cevallos, 2011).

La estadística experimental en el mundo moderno es una necesidad real, está presente en todas las áreas del conocimiento humano como una herramienta para auxiliar las decisiones a ser tomadas, permitiendo mayor seguridad a la investigación a ser transformada en tecnología y utilizada por la humanidad o por parte de ella (López et al., 2006).

El presente trabajo tiene como objetivo determinar la existencia de diferencias estadísticas significativas en las distribuciones del espesor del sistema dúplex (Fe/Zn/Pintura), antes y después de realizado el ensayo de humedad y temperatura 
con condensación en atmósfera constante, a partir de diferentes pruebas realizadas en el software Statgraphics Centurion. Las pinturas que forman parte del sistema dúplex son las mismas, solo varían su color.

\section{MATERIALES Y MÉTODOS}

\subsection{Ensayo climático acelerado de humedad y temperatura controlada}

Para cumplir el objetivo de esta investigación se utiliza la cámara climática de humedad y temperatura con condensación en atmósfera constante modelo DYCOMETAL VCK-300, la cual presenta una temperatura de $40 \pm 2{ }^{\circ} \mathrm{C}$ y humedad relativa entre 99 y 100\% (NC-ISO-6270-2, 2017).

\section{Procedimiento}

- La cubeta del fondo se debe llenar con agua de manera que la profundidad no sea inferior a $10 \mathrm{~mm}$, en ningún momento del ensayo. Además no se deben exponer a la vez recubrimientos que puedan ejercer una influencia entre ellos.

- Las probetas de ensayo se deben colocar en la cámara con un ángulo igual o mayor a $60^{\circ}$ respecto a la horizontal de manera que no se encuentren en contacto unas con otras y puedan radiar calor adecuadamente.

- Se debe tener cuidado para asegurar que la condensación de las paredes y el techo de la cámara climática no gotee sobre las probetas de ensayo, ni de unas probetas a otras.

- Se exponen las probetas a un período de $1000 \mathrm{~h}$.

\subsection{Determinación del espesor del recubrimiento sobre sustrato magnético}

Se emplea el método de medición no destructiva del espesor de recubrimientos no conductores sobre base magnético y se utiliza el medidor modelo HUATEC TG 2100 FN, incorporándose el principio de atracción magnética existente entre el imán y el metal base, influenciada por la presencia del recubrimiento, es decir, la reluctancia de un flujo magnético pasando a través del recubrimiento y el metal base (ISO-2178, 2016).

El espesor del revestimiento de cada pieza a controlar se medirá sobre la superficie significativa, y se tomaron 3 puntos de medida (alrededor de $1 \mathrm{~cm}^{2}$ ), procediéndose a 5 lecturas individuales del espesor en cada punto seleccionado. Fueron realizadas un total de 120 mediciones en las cuatro probetas analizadas. La metodología seguida se presenta en la Fig. 1.

El material utilizado en la realización de los ensayos se corresponde a láminas de acero al carbono de baja aleación laminado en caliente, recubierto por ambas caras por un recubrimiento de zinc con espesores que oscilan en un intervalo entre 16 y $19 \mu \mathrm{m}$ y presentan una capa de pintura electrostática en polvo. Las placas rectangulares utilizadas presentan dimensiones de $0,1 \times 0,15 \mathrm{~m}$.

\section{"s sargraphics}
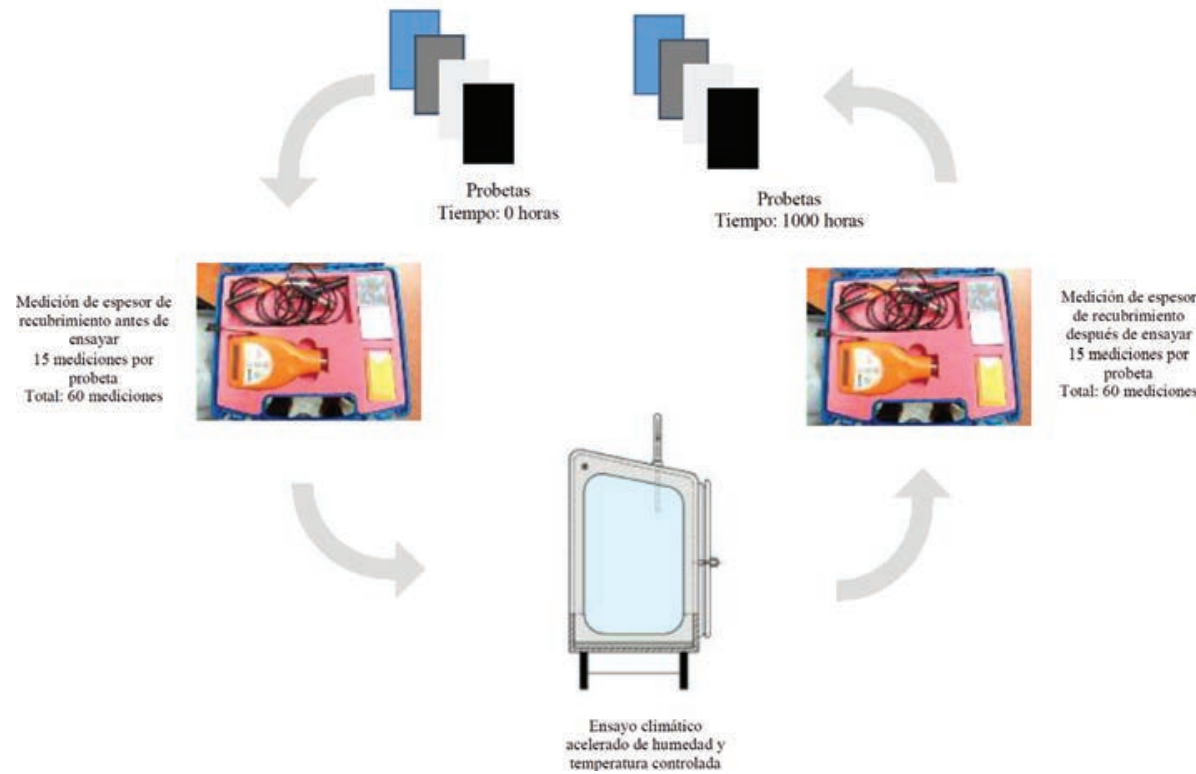
15 mediciones por probeta
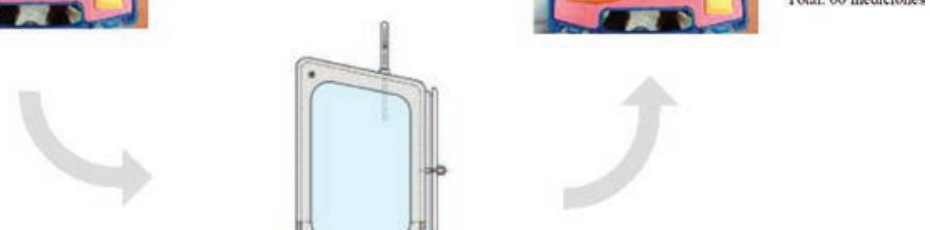

Figura 1. Metodología empleada para el estudio propuesto. 
Las probetas serán identificadas con la siguiente nomenclatura:

$\begin{array}{ll}- & \text { M1: RAL } 7016 \\ - & \text { M2: RAL } 7040 \\ - & \text { M3: RAL } 9002 \\ - & \text { M4: RAL } 7037\end{array}$

\subsection{Análisis estadístico}

Para la realización del análisis estadístico se utiliza el software Statgraphics Centurion XVII, el cual permite la evaluación de los resultados obtenidos en el ensayo de espesor del recubrimiento, a partir de una comparación entre las medias y desviaciones estándares teniendo en cuenta los valores obtenidos de espesor de recubrimiento en las cuatro probetas, antes y después de ser sometidas al ensayo climático acelerado de humedad y temperatura controlada.

\section{Procedimiento}

- Se introducen los valores obtenidos de espesor en las columnas correspondientes y se selecciona la opción: Comparar/ Dos muestras/ Muestras independientes. En este caso el software Statgraphics Centurion define las muestras como las distribuciones de los valores del muestreo antes y después del ensayo acelerado.

- En la ventana tablas y gráficos, se debe seleccionar las opciones siguientes: resumen del análisis, resumen estadístico, comparación de medias, comparación de desviaciones estándar, histograma y gráfico de caja y bigotes.

\section{RESULTADOS}

\subsection{Resistencia al ensayo climático acelerado de humedad y temperatura controlada}

Se colocaron las cuatro probetas en la cámara de humedad y temperatura con condensación en atmósfera constante durante un tiempo de exposición de 1000 h y no se observa la presencia de defectos que denoten la degradación del recubrimiento tales como: ampollamiento (NC-ISO-4628-2, 2018), oxidación (NC-ISO-4628-3, 2018), agrietamiento (NC-ISO-4628-4, 2018), descamación (NC-ISO-4628-5, 2018) ni grado de enyesado (NC-ISO-4628-6, 2018). Se comprueba la resistencia del sistema de protección utilizado en el acero ante condiciones severas de temperatura y humedad.

\subsection{Determinación de los espesores de recubrimientos sobre sustratos magnéticos}

Antes de ubicar las probetas en la cámara climática, se determinaron teniendo en cuenta el procedimiento, los valores mínimos, máximos, desviación estándar, promedios del recubrimiento analizado en el estudio. Se presentan en las Tablas 1 y 2 los resultados obtenidos antes y después de ser sometidas a ensayo acelerado.

\subsection{Análisis estadístico}

El procedimiento utilizado se basa en una comparación entre las medias y desviaciones estándares teniendo en cuenta los valores obtenidos de espesor de recubrimiento en las cuatro probetas, antes y después de ser sometidas al ensayo climático acelerado de humedad y temperatura controlada.

TAbla 1. Mediciones de espesor de recubrimiento en las muestras ensayadas antes de ser sometidas a ensayo climático acelerado

\begin{tabular}{|c|c|c|c|c|c|c|}
\hline \multirow[b]{2}{*}{ Probeta } & \multirow[b]{2}{*}{ Puntos } & \multicolumn{5}{|c|}{ Espesor de recubrimiento $(\mu \mathrm{m})$} \\
\hline & & Medición 1 & Réplica 1 & Réplica 2 & Réplica 3 & Réplica 4 \\
\hline \multirow[t]{3}{*}{ M1 } & 1 & 54,8 & 55,1 & 54,2 & 53,1 & 51,6 \\
\hline & 2 & 53,8 & 54,7 & 54,1 & 55,2 & 53,9 \\
\hline & 3 & 53,9 & 54,1 & 52,4 & 53,4 & 53,0 \\
\hline \multirow[t]{3}{*}{ M2 } & 1 & 41,5 & 43,3 & 42,4 & 42,2 & 43,0 \\
\hline & 2 & 41,4 & 42,9 & 41,4 & 42,5 & 42,9 \\
\hline & 3 & 42,8 & 42,8 & 43,1 & 41,9 & 42,1 \\
\hline \multirow[t]{3}{*}{ M3 } & 1 & 45,2 & 47,7 & 45,9 & 46,2 & 46,0 \\
\hline & 2 & 47,0 & 45,0 & 44,9 & 48,6 & 46,5 \\
\hline & 3 & 45,2 & 48,2 & 44,3 & 46,6 & 47,5 \\
\hline \multirow[t]{3}{*}{ M4 } & 1 & 51,7 & 52,0 & 52,5 & 51,8 & 55,8 \\
\hline & 2 & 51,2 & 51,5 & 51,8 & 51,9 & 52,3 \\
\hline & 3 & 53,0 & 52,1 & 53,5 & 55,2 & 52,7 \\
\hline
\end{tabular}


TABLA 2. Mediciones de espesor de recubrimiento en las muestras ensayadas después de ser sometidas a ensayo climático acelerado

\begin{tabular}{lcccccc}
\hline & & \multicolumn{5}{c}{ Espesor de recubrimiento $(\boldsymbol{\mu m})$} \\
\cline { 2 - 6 } Probeta & Puntos & Medición 1 & Réplica 1 & Réplica 2 & Réplica 3 & Réplica 4 \\
\hline M1 & 1 & 52,5 & 52,3 & 50,9 & 52,0 & 50,4 \\
& 2 & 52,3 & 52,6 & 50,0 & 51,0 & 50,2 \\
& 3 & 52,0 & 50,1 & 51,0 & 50,2 & 50,0 \\
M2 & 1 & 41,1 & 39,8 & 40,7 & 40,3 & 41,0 \\
& 2 & 40,6 & 41,0 & 40,6 & 39,4 & 38,5 \\
& 3 & 41,1 & 40,4 & 39,1 & 39,9 & 39,1 \\
M3 & 1 & 45,2 & 43,9 & 45,5 & 45,1 & 44,6 \\
& 2 & 44,8 & 44,8 & 43,4 & 43,5 & 45,0 \\
& 3 & 45,3 & 43,7 & 44,7 & 43,5 & 43,1 \\
& 1 & 49,0 & 49,5 & 50,2 & 50,0 & 49,6 \\
& 2 & 49,9 & 50,4 & 50,7 & 50,0 & 50,6 \\
\end{tabular}

\subsubsection{Para probeta M1 (RAL 7016)}

En la Tabla 3 se presenta el resumen estadístico para las dos muestras de datos. Los valores del sesgo estandarizado y la curtosis estandarizada se encuentran dentro del rango esperado de -2 a 2 , indicando que los datos provienen de una distribución normal.

\section{- Comparación de medias}

La prueba $t$ se construye para determinar la existencia de diferencias entre las medias. Puesto que el valor-P $(6,84852 \mathrm{E}-8)$ calculado es menor que 0,05 , se puede rechazar la hipótesis nula en favor de la alterna para alfa $=0,05$. Prueba t para comparar las medias:

- Hipótesis nula: media $_{1}=$ media $_{2}$

- Hipótesis alternativa: media $_{1} \neq$ media $_{2}$

- Comparación de desviaciones estándares

La prueba $\mathrm{F}$ construye para determinar la existencia de diferencias entre las desviaciones estándar. Puesto que el valor-P $(0,965685)$ calculado no es menor que 0,05 , no se puede rechazar la hipótesis nula, con un nivel de confianza del 95,0\%. Prueba F para comparar las desviaciones estándares:

- Hipótesis Nula: $\operatorname{sigma}_{1}=$ sigma $_{2}$

- Hipótesis Alternativa.: sigma $_{1} \neq$ sigma $_{2}$

- Prueba Kolmogorov-Smirnov

Esta prueba se realiza calculando la distancia máxima entre las distribuciones acumuladas de las dos muestras. En este caso, la distancia máxima es 0,866667 . Debido a que el valor-P $(0,0000255846)$
TABLA 3. Resumen estadístico obtenido para la probeta M1

\begin{tabular}{lcc}
\hline & Espesor $\mathbf{0 ~ h}$ & Espesor $\mathbf{1 0 0 0} \mathbf{~ h}$ \\
\hline Recuento & 15 & 15 \\
Promedio & 53,82 & 51,1667 \\
Desviación Estándar & 0,996566 & 1,0083 \\
Coeficiente de Variación & $1,85166 \%$ & $1,97062 \%$ \\
Mínimo & 51,6 & 50,0 \\
Máximo & 55,2 & 52,6 \\
Rango & 3,6 & 2,6 \\
Sesgo Estandarizado & $\mathbf{- 1 , 1 0 0 1 1}$ & $\mathbf{0 , 3 4 4 3 6}$ \\
Curtosis Estandarizada & $\mathbf{0 , 2 7 6 7 5 9}$ & $\mathbf{- 1 , 4 0 4 4 3}$ \\
\hline
\end{tabular}

es menor que 0,05 , existe una diferencia estadísticamente significativa entre las dos distribuciones con un nivel de confianza del 95,0\%.

\section{- Histograma de Frecuencias}

La Fig. 2 muestra un gráfico de barras, el cual ilustra el número de observaciones en un grupo de intervalos que abarca el rango de los datos recogidos. Se presenta una comparación de las dos distribuciones simultáneamente. El histograma para los valores de espesor después de ser sometidas las probetas al ensayo climático acelerado, se encuentra por debajo de la línea horizontal, con un desplazamiento hacia la izquierda.

\section{- Gráfico de Caja y Bigotes}

La Fig. 3 muestra un gráfico de Caja y Bigotes, el cual presenta una caja central que cubre la mitad de las observaciones en cada distribución. Los bigotes se extienden entre los valores máximo y mínimo de 


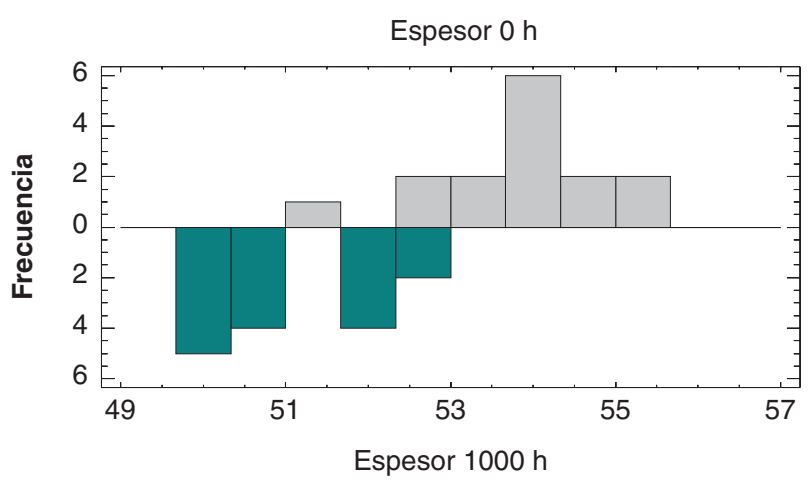

Figura 2. Histograma de frecuencias dual para la probeta M1.

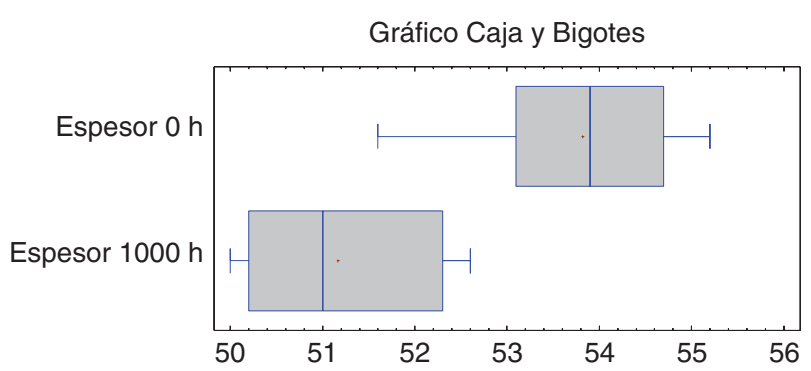

Figura 3. Gráfico dual de Caja y Bigotes para la probeta M1.

cada muestra, excepto para algunos puntos que se sitúan inusualmente lejanos a las cajas. Se dibuja una línea vertical en cada caja a la altura de la mediana, mientras que los pequeños signos más situados en el interior de las cajas indican las localizaciones de las medias muestrales.

Se evidencia un aparente desvío del centro de la distribución hacia la izquierda. El intervalo cubierto por los valores de espesor obtenidos después del ensayo es más ancho que el intervalo cubierto por los valores de espesor obtenidos antes del ensayo.

\subsubsection{Para probeta M2 ( $R A L 7040)$}

En la Tabla 4 se presenta el resumen estadístico para las dos muestras de datos. Los valores del sesgo estandarizado y la curtosis estandarizada se encuentran dentro del rango esperado de -2 a 2 , indicando que los datos provienen de una distribución normal.

\section{- Comparación de medias}

La prueba $t$ se construye para determinar la existencia de diferencias entre las medias. Puesto que el valor-P $(5,26257 \mathrm{E}-9)$ calculado es menor que 0,05 , se puede rechazar la hipótesis nula en favor de la alterna para alfa $=0,05$. Prueba t para comparar las medias:

- Hipótesis nula: media $_{1}=$ media $_{2}$

- Hipótesis alternativa: media $_{1} \neq$ media $_{2}$
TABLA 4. Resumen estadístico obtenido para la probeta M2

\begin{tabular}{lcc}
\hline & Espesor 0 h & Espesor 1000 h \\
\hline Recuento & 15 & 15 \\
Promedio & 42,4133 & 40,1733 \\
Desviación Estándar & 0,636808 & 0,832781 \\
Coeficiente de Variación & $1,50143 \%$ & $2,07297 \%$ \\
Mínimo & 41,4 & 38,5 \\
Máximo & 43,3 & 41,1 \\
Rango & 1,9 & 2,6 \\
Sesgo Estandarizado & $\mathbf{- 0 , 7 3 1 2 6 1}$ & $\mathbf{- 1 , 0 5 0 7 5}$ \\
Curtosis Estandarizada & $\mathbf{- 0 , 8 6 5 8 4 9}$ & $\mathbf{- 0 , 5 5 3 6 5 5}$ \\
\hline
\end{tabular}

\section{- Comparación de desviaciones estándares}

La prueba $F$ construye para determinar la existencia de diferencias entre las desviaciones estándar. Puesto que el valor-P $(0,326874)$ calculado no es menor que 0,05 , no se puede rechazar la hipótesis nula, con un nivel de confianza del $95,0 \%$. Prueba $F$ para comparar las desviaciones estándares:

- Hipótesis Nula: $\operatorname{sigma}_{1}=\operatorname{sigma}_{2}$

- Hipótesis Alternativa.: $\operatorname{sigma}_{1} \neq$ sigma $_{2}$

\section{- Prueba Kolmogorov-Smirnov}

Esta prueba se realiza calculando la distancia máxima entre las distribuciones acumuladas de las dos muestras. En este caso, la distancia máxima es 1,0 . Debido a que el valor-P $(6,11805 \mathrm{E}-7)$ es menor que 0,05 , existe una diferencia estadísticamente significativa entre las dos distribuciones con un nivel de confianza del $95,0 \%$.

\section{- Histograma de Frecuencias}

La Fig. 4 muestra un gráfico de barras, el cual ilustra el número de observaciones en un grupo de intervalos que abarca el rango de los datos recogidos. Se presenta una comparación de las dos distribuciones simultáneamente. El histograma para los valores de espesor después de ser sometidas las probetas al ensayo climático acelerado, se encuentra por debajo de la línea horizontal, con un desplazamiento hacia la izquierda.

\section{- Gráfico de Caja y Bigotes}

La Fig. 5 muestra un aparente desvío del centro de la distribución hacia la izquierda. El intervalo cubierto por los valores de espesor obtenidos después del ensayo es más ancho que el intervalo cubierto por los valores de espesor obtenidos antes del ensayo. 


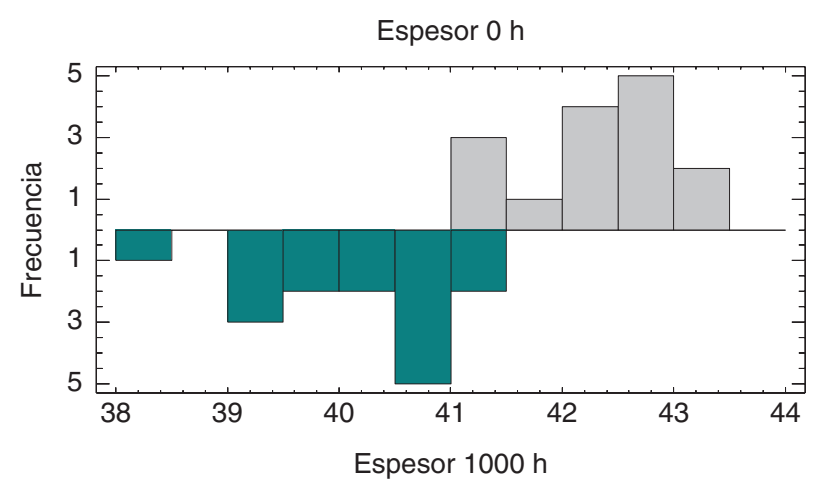

FIgURA 4. Histograma de frecuencias dual para la probeta M2.

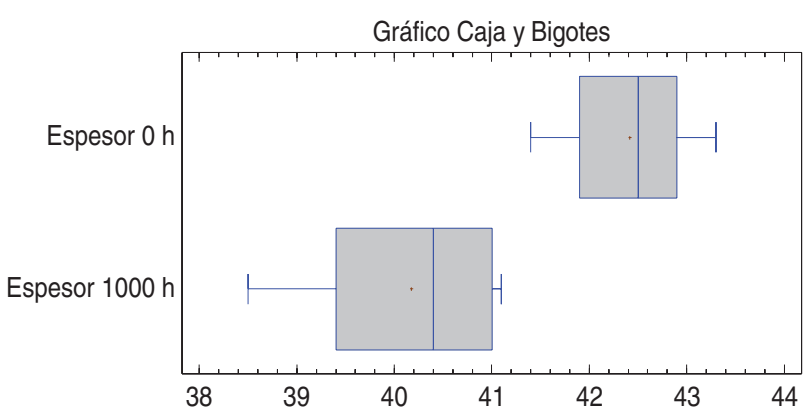

Figura 5. Gráfico dual de Caja y Bigotes para la probeta M2.

\subsubsection{Para probeta M3 (RAL 9002)}

En la Tabla 5 se presenta el resumen estadístico para las dos muestras de datos. Los valores del sesgo estandarizado y la curtosis estandarizada se encuentran dentro del rango esperado de -2 a 2 , indicando que los datos provienen de una distribución normal.

\section{- Comparación de medias}

La prueba $t$ se construye para determinar la existencia de diferencias entre las medias. Puesto que el valor-P $(0,000038276)$ calculado es menor que 0,05 , se puede rechazar la hipótesis nula en favor de la alterna para alfa $=0,05$. Prueba t para comparar las medias:

- Hipótesis nula: media $_{1}=$ media $_{2}$

- Hipótesis alternativa: media $_{1} \neq$ media $_{2}$

\section{- Comparación de desviaciones estándares}

La prueba $\mathrm{F}$ construye para determinar la existencia de diferencias entre las desviaciones estándar. Puesto que el valor-P $(0,0870233)$ calculado no es menor que 0,05 , no se puede rechazar la hipótesis
TABLA 5. Resumen estadístico obtenido para la probeta M3

\begin{tabular}{lcc}
\hline & Espesor $\mathbf{0 ~ h}$ & Espesor $\mathbf{1 0 0 0} \mathbf{~ h}$ \\
\hline Recuento & 15 & 15 \\
Promedio & 46,32 & 44,4067 \\
Desviación Estándar & 1,28852 & 0,802199 \\
Coeficiente de Variación & $2,78178 \%$ & $1,80648 \%$ \\
Mínimo & 44,3 & 43,1 \\
Máximo & 48,6 & 45,5 \\
Rango & 4,3 & 2,4 \\
Sesgo Estandarizado & $\mathbf{0 , 4 1 3 3 7 6}$ & $\mathbf{- 0 , 5 0 7 1 8 2}$ \\
Curtosis Estandarizada & $\mathbf{- 0 , 7 0 3 0 4 3}$ & $\mathbf{- 1 , 2 0 0 3 6}$ \\
\hline
\end{tabular}

\section{Espesor $0 \mathrm{~h}$}

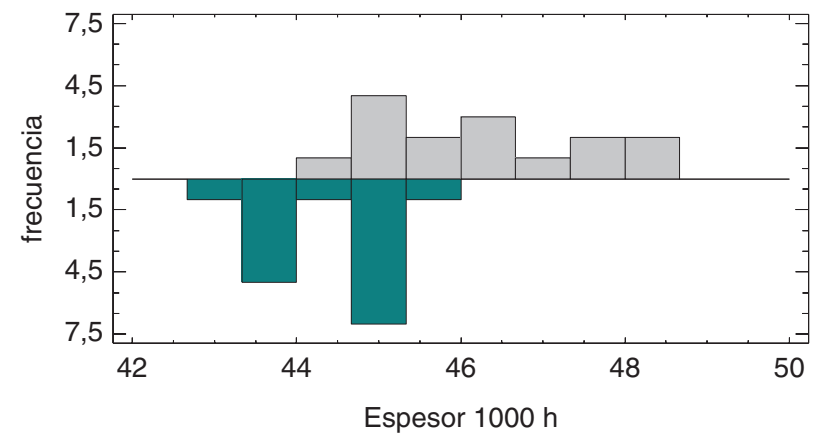

FIGURA 6. Histograma de frecuencias dual para la probeta M3.

nula, con un nivel de confianza del 95,0\%. Prueba F para comparar las desviaciones estándares:

- Hipótesis Nula: sigma $_{1}=$ sigma $_{2}$

- Hipótesis Alternativa.: sigma $_{1} \neq$ sigma $_{2}$

\section{- Prueba Kolmogorov-Smirnov}

Esta prueba se realiza calculando la distancia máxima entre las distribuciones acumuladas de las dos muestras. En este caso, la distancia máxima es 1,0 . Debido a que el valor-P $(0,00254527)$ es menor que 0,05 , existe una diferencia estadísticamente significativa entre las dos distribuciones con un nivel de confianza del $95,0 \%$.

\section{- Histograma de Frecuencias}

La Fig. 6 muestra un gráfico de barras, el cual ilustra el número de observaciones en un grupo de intervalos que abarca el rango de los datos recogidos. Se presenta una comparación de las dos distribuciones simultáneamente. El histograma para los valores de espesor después de ser sometidas las probetas al ensayo climático acelerado, se encuentra por debajo de la línea horizontal, con un desplazamiento hacia la izquierda. 


\section{- Gráfico de Caja y Bigotes}

La Fig. 7 muestra un aparente desvío del centro de la distribución hacia la izquierda. El intervalo cubierto por los valores de espesor obtenidos antes del ensayo es más ancho que el intervalo cubierto por los valores de espesor obtenidos después del ensayo.

\subsubsection{Para probeta M4 (RAL 7037)}

En la Tabla 6 se presenta el resumen estadístico para las dos muestras de datos. Los valores del sesgo estandarizado y la curtosis estandarizada se encuentran dentro del rango esperado de -2 a 2 , indicando que los datos provienen de una distribución normal.

\section{- Comparación de medias}

La prueba $t$ se construye para determinar la existencia de diferencias entre las medias. Puesto que el valor-P $(3,27298 \mathrm{E}-10)$ calculado es menor que 0,05 , se puede rechazar la hipótesis nula en favor de la alterna para alfa $=0,05$. Prueba t para comparar las medias:

- Hipótesis nula: media $_{1}=$ media $_{2}$

- Hipótesis alternativa: media $_{1} \neq$ media $_{2}$

Comparación de desviaciones estándares

La prueba $F$ construye para determinar la existencia de diferencias entre las desviaciones estándar. Puesto que el valor-P $(0,510103)$ calculado no es menor que 0,05 , no se puede rechazar la hipótesis

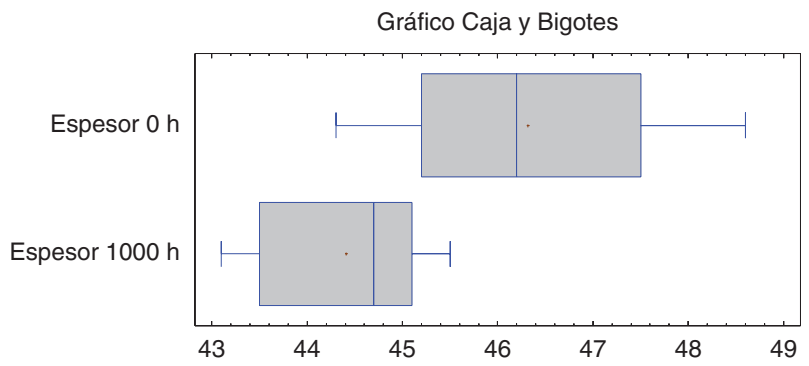

FIgura 7. Gráfico dual de Caja y Bigotes para la probeta M3.

TABLA 6. Resumen estadístico obtenido para la probeta M4

\begin{tabular}{lcc}
\hline & Espesor $\mathbf{0 ~ h}$ & Espesor $\mathbf{1 0 0 0} \mathbf{~ h}$ \\
\hline Recuento & 15 & 15 \\
Promedio & 52,04 & 50,2267 \\
Desviación Estándar & 0,476295 & 0,570046 \\
Coeficiente de Variación & $0,915248 \%$ & $1,13495 \%$ \\
Mínimo & 51,2 & 49,0 \\
Máximo & 53,1 & 51,2 \\
Rango & 1,9 & 2,2 \\
Sesgo Estandarizado & $\mathbf{0 , 9 2 1 3 1 7}$ & $\mathbf{- 0 , 8 3 1 6 9 2}$ \\
Curtosis Estandarizada & $\mathbf{0 , 5 8 0 0 6 6}$ & $\mathbf{0 , 1 5 9 6 1 9}$ \\
\hline
\end{tabular}

nula, con un nivel de confianza del 95,0\%. Prueba F para comparar las desviaciones estándares:

- Hipótesis Nula: sigma $_{1}=$ sigma $_{2}$

- Hipótesis Alternativa.: sigma $_{1} \neq$ sigma $_{2}$

Prueba Kolmogorov-Smirnov

Esta prueba se realiza calculando la distancia máxima entre las distribuciones acumuladas de las dos muestras. En este caso, la distancia máxima es 1,0 . Debido a que el valor-P $(6,11805 \mathrm{E}-7)$ es menor que 0,05 , existe una diferencia estadísticamente significativa entre las dos distribuciones con un nivel de confianza del 95,0\%.

\section{- Histograma de Frecuencias}

La Fig. 8 muestra un gráfico de barras, el cual ilustra el número de observaciones en un grupo de intervalos que abarca el rango de los datos recogidos. Se presenta una comparación de las dos distribuciones simultáneamente. El histograma para los valores de espesor después de ser sometidas las probetas al ensayo climático acelerado, se encuentra por debajo de la línea horizontal, con un desplazamiento hacia la izquierda.

\section{- Gráfico de Caja y Bigotes}

La Fig. 9 muestra un aparente desvío del centro de la distribución hacia la izquierda. El intervalo cubierto por los valores de espesor obtenidos después

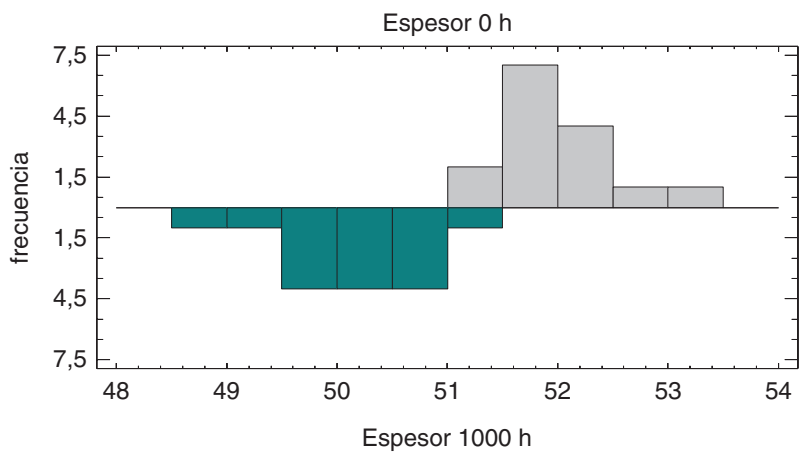

FIGURA 8. Histograma de frecuencias dual para la probeta M4.

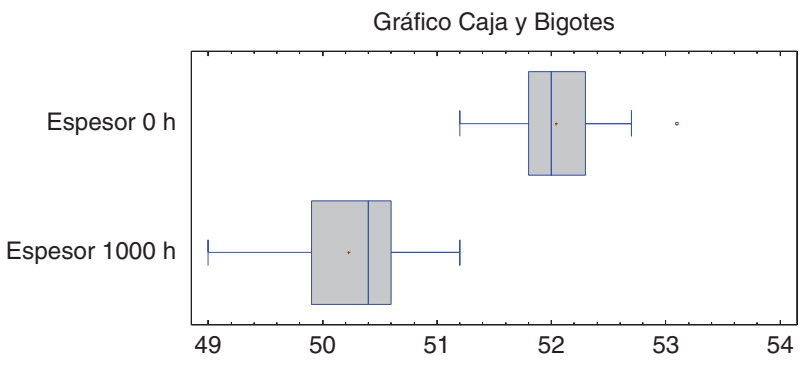

Figura 9. Gráfico dual de Caja y Bigotes para la probeta M4. 
del ensayo es más ancho que el intervalo cubierto por los valores de espesor obtenidos antes del ensayo.

\section{CONCLUSIONES}

Se demostró la influencia de la acción de la humedad y temperatura con condensación en atmósfera constante, sobre el espesor de recubrimiento del sistema dúplex ( $\mathrm{Fe} / \mathrm{Zn}$ /Pintura), a partir de las diferentes pruebas realizadas con el software Statgraphics Centurion.

- Los datos reportados de espesor de recubrimiento provienen de las distribuciones normales, puesto que los valores del sesgo estandarizado y la curtosis estandarizada se encuentran dentro del rango esperado de -2 a 2.

- La existencia de diferencias significativas entre las medias, puesto que el valor-P calculado en cada caso analizado, es menor que 0,05 , aceptándose la hipótesis alternativa para alfa de 0,05.

- La no existencia diferencias entre las desviaciones estándares, puesto que el valor-P en cada uno de los casos es superior a 0,05 , aceptándose la hipótesis nula con un nivel de confianza del $95,0 \%$.

- A partir de la prueba de Kolmogorov-Smirnov se demuestra la existencia de una diferencia estadísticamente significativa entre las distribuciones acumuladas con un nivel de confianza del $95,0 \%$.

- Se demuestra una tendencia a la disminución del espesor de recubrimiento en las condiciones prefijadas a partir del histograma de frecuencia y diagrama de Caja y Bigotes.

A partir de los análisis realizados se pudo demostrar la influencia del ensayo escogido en la variación del espesor de recubrimiento, encontrándose los mismos en un intervalo adecuado para garantizar la resistencia a la corrosión, pues no se evidencio deterioro visible en la superficie de las probetas analizadas.

\section{AGRADECIMIENTOS}

Este trabajo se realizó con el apoyo del Laboratorio de Ensayos de Tropicalización LABET, perteneciente al Centro de Tecnología y Calidad del Ministerio de Industrias, Cuba.

\section{REFERENCIAS}

Castañeda, A., Rivero, C., Corvo, F. (2012). Evaluación de sistemas de protección contra la corrosión en la rehabilitación de estructuras construidas en sitios de elevada agresividad corrosiva en Cuba. Revista de la Construcción 11 (3), 49-61. https://doi.org/10.4067/S0718-915X2012000300005.

CITMA (1997). Ley 81 del Medio Ambiente, 7 Stat. http://www. sld.cu/galerias/pdf/sitios/insat/ley-81-citma.pdf.

Cutipa Mamani, C.A. (2014). Planteamiento para determinar comportamientos óptimos entre sistemas de protección dúplex (Galvanizado en caliente/frío + recubrimiento) para estructuras metálicas de SE y LT. Facultad de Ingeniería de Procesos, UNSA. http://repositorio.unsa.edu.pe/ handle/UNSA/2908.

ISO 2178 (2016). Non-magnetic coatings on magnetic substrates - Measurement of coating thickness - Magnetic method. International Organization for Standardization, page 37.

López, C.M., Iturralde, M.A., Claro, R., Ruiz, L., Cabrera, G.J., Molerio, L., Rodríguez, Y. (2006). Introducción al conocimiento del medio ambiente. Vol. 1, Editorial-Académica, pp. 1-32. http://aulag.aulas.rimed.cu/files/2013/11/ Tabloide-Medio-Ambiente.pdf.

Marrero-Águila, R., González Prada, C., González-Ortega, L., McNeil Montañés, B. (2013). Ensayos de corrosión naturales y acelerados en sistemas de pinturas aplicados sobre acero. Tecnología Ouímica 33 (3), 233-241.

Mina Cevallos, E. (2011). Ensayos de corrosión metálica en laboratorio y su correlación con ensayos de campo. Tesis Ing. Química (IQUIM), Escuela Politécnica Nacional Quito, Ed. QUITO/EPN.

NC-ISO 6270-2 (2017). Pinturas y barnices. Determinación de la resistencia a la humedad. Parte 2: Condensación (exposición en cabina con depósito de agua caliente).

NC-ISO-4628-2 (2018). Pinturas y barnices-Evaluación de la degradación de los recubrimientos de pintura. Designación de la intensidad, cantidad y tamaño de los tipos más comunes de defectos-Parte 2: Designación del grado de ampollamiento.

NC-ISO-4628-3 (2018). Pinturas y barnices - Evaluación de la degradación de los recubrimientos - Designación de la intensidad, cantidad y tamaño de los tipos más comunes de defectos - Parte 3: Evaluación del grado de oxidación.

NC-ISO-4628-4 (2018). Pinturas y barnices - Evaluación de la degradación de los recubrimientos - Designación de la intensidad, cantidad y tamaño de los tipos más comunes de defectos - Parte 4: Evaluación del grado de agrietamiento.

NC-ISO-4628-5 (2018). Pinturas y barnices - Evaluación de la degradación de los recubrimientos - Designación de la intensidad, cantidad y tamaño de los tipos más comunes de defectos - Parte 5: Evaluación del grado de descamación.

NC-ISO-4628-6 (2018). Pinturas y barnices - Evaluación de la degradación de los recubrimientos - Designación de la intensidad, cantidad y tamaño de los tipos más comunes de defectos - Parte 6: evaluación del grado de enyesado por el método de la cinta.

Simancas Peco, J. (1992). Estudio de distintas variables con influencia en el comportamiento en la atmósfera de los recubrimientos anticorrosivos aplicados sobre el acero. Tesis Doctoral, Universidad Complutense de Madrid, Madrid.

Simancas, J., Morcillo, M. (1998). Factores condicionantes de la durabilidad de los sistemas de pinturas anticorrosivas sobre acero en exposiciones atmosféricas. Rev. Metal. 34 (Extra), 132-136. https://doi.org/10.3989/revmetalm.1998. v34.iExtra.725.

Vásquez Rodríguez, C.A. (2013). Caracterización del comportamiento de corrosión en pruebas aceleradas para acero galvanizado y pintado. Maestría Tesis, Universidad Autónoma de Nuevo León, San Nicolás de los Garza. http:// eprints.uanl.mx/id/eprint/3800. 